\title{
Pairing of Spermatozoa in the Epididymis of the Opossum (Didelphis virginiana): A Scanning Electron Microscopic Study
}

\author{
William J. Krause and J. Harry Cutts \\ Department of Anatomy, University of Missouri School of Medicine, Columbia, U. S. A.
}

Received October 11, 1978

Summary. Spermatozoa of the opossum, Didelphis virginiana, were taken from three different regions of the epididymis and examined by scanning electron microscopy, phasecontrast and dark-field microscopy. It has been demonstrated that marked morphological changes occur in spermatozoa during their passage through the epididymis. In the first segment or head of the epididymis, spermatozoa are non-motile and the long axis of their nuclei is perpendicular to that of the tail. The nucleus is $V$-shaped with a thick and a thin arm. At the junction of the two arms, where the arms join, the articular fossa receives the capitulum of the connecting piece which attaches the head of the spermatozoon to the tail. Spermatozoa from the central region of the epididymis show a re-orientation of the nuclei which now lie parallel to the long axis of the tail. Both paired and unpaired spermatozoa are found and show an increase in motility. In the caudal region of the epididymis all spermatozoa are paired. Pairing occurs as a result of the close apposition of adjacent cell membranes covering the acrosomes of the larger arms.

Pairing of spermatozoa of the North American opossum, Didelphis virginiana, was reported first by SELENKA (1887) who observed that sperm recovered from the vaginas of recently bred females, swam in closely united pairs. Similar pairing of spermatozoa has been found to occur in all of the South American marsupials studied to date, but has not been observed in the Austral-Asian species (Biggers and DeLamater, 1965; Hughes, 1965; Phillips, 1972). Since the initial observation of sperm-pairing in Didelphis, it has been shown that the pairing occurs in the epididymis and not in the testis (Retzius, 1909; Jordan, 1911; Duesberg, 1920; McCrady, 1938; Biggers and CREed, 1962; Phillips, 1974). As the spermatozoa of the opossum pass through the epididymis, a very close association develops between the cell membranes of the heads of the spermatozoa in the regions of the acrosomes, but morphological junctions are not observed between the pairing heads (PHILlips, 1974). Flagella of the paired spermatozoa beat in a co-ordinated, alternating manner (PHILLIPS, 1972).

The present report describes the morphological appearances of the spermatozoa of the opossum, Didelphis virginiana, as seen by the light and scanning electron microscope and presents details of the pairing as it occurs in different regions of the epididymis. 

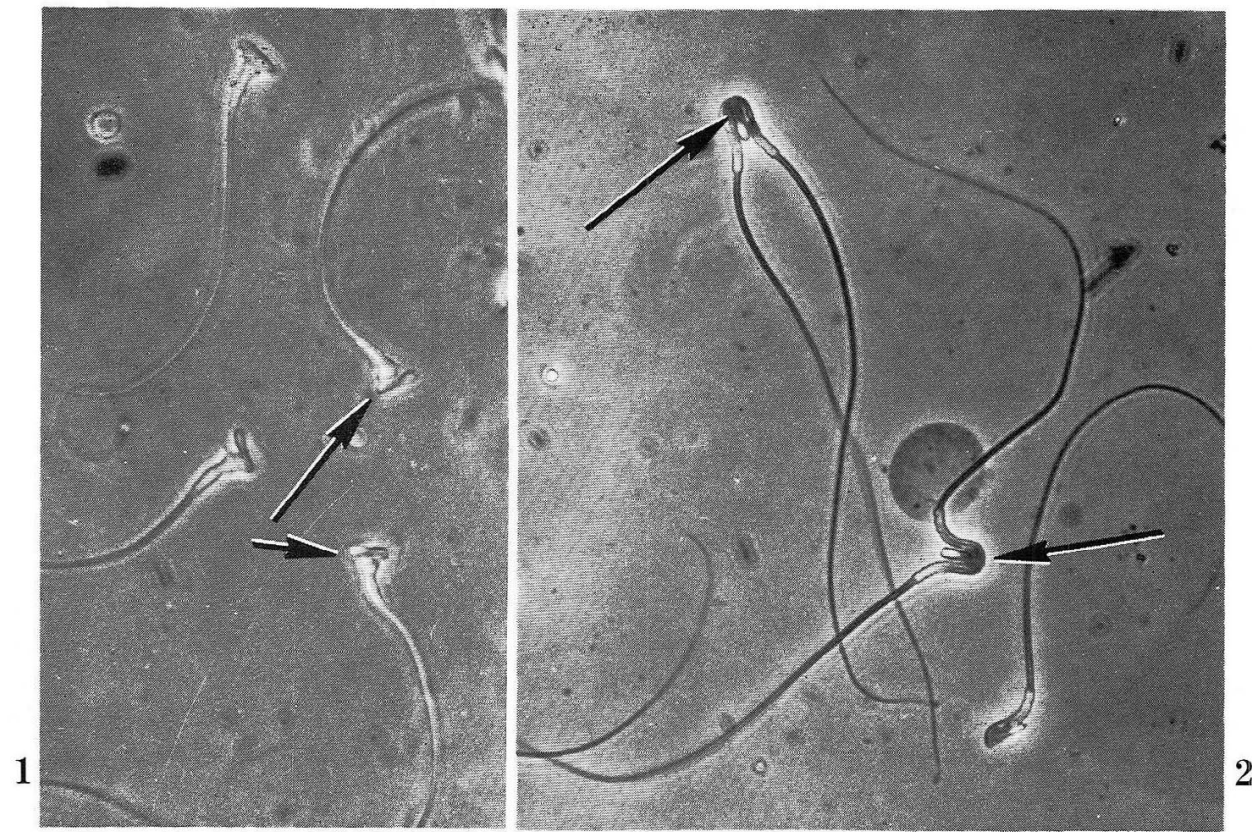

Fig. 1. Spermatozoa from the first (head) region of the epididymis. Note that the flattened nuclei (arrows) lie perpendicular to the long axis of the tail. Phase contrast. $\times 400$

Fig. 2. Five spermatozoa, four of which are paired (arrows), from the central region of the epididymis. Note the change in position of the nucleus of the unpaired spermatozoon as compared to those shown in Figure 1 . Phase contrast. $\times 400$

\section{MATERIALS AND METHODS}

Spermatozoa from the epididymides of seven adult opossums were examined. The animals were killed by ether anesthesia, the scrotal sac was quickly opened and the testes and epididymides removed. The head, body and tail regions of the epididymis were identified, separated from each other and minced with sissors in Kreb's buffer. Samples of spermatozoa from each region were placed on glass slides, cover-slipped, and observed with phase-contrast and dark-field microscopy. Additional aliquots of spermatozoa from each of the three regions were placed in $0.1 \%$ glutaraldehyde in Kreb's buffer ( $\mathrm{pH}$ 7.4). The spermatozoa were osmicated in $1.33 \% \mathrm{OsO}_{4}$ in buffer for $1 \mathrm{hr}$ according to ARNOLD et al. (1971), dehydrated in alcohol, transferred to amyl acetate and pipetted onto small pieces of aluminum foil to be critical point-dried with liquid $\mathrm{CO}^{2}$. The aluminum foil strips containing the spermatozoa were then placed on spinner stubs and coated with gold-palladium to a depth of $20 \mathrm{~nm}$ in a vacuum evaporator. Specimens were viewed in a Cambridge Stereoscan Mark II electron microscope at $20 \mathrm{kV}$ of accelerating voltage. 


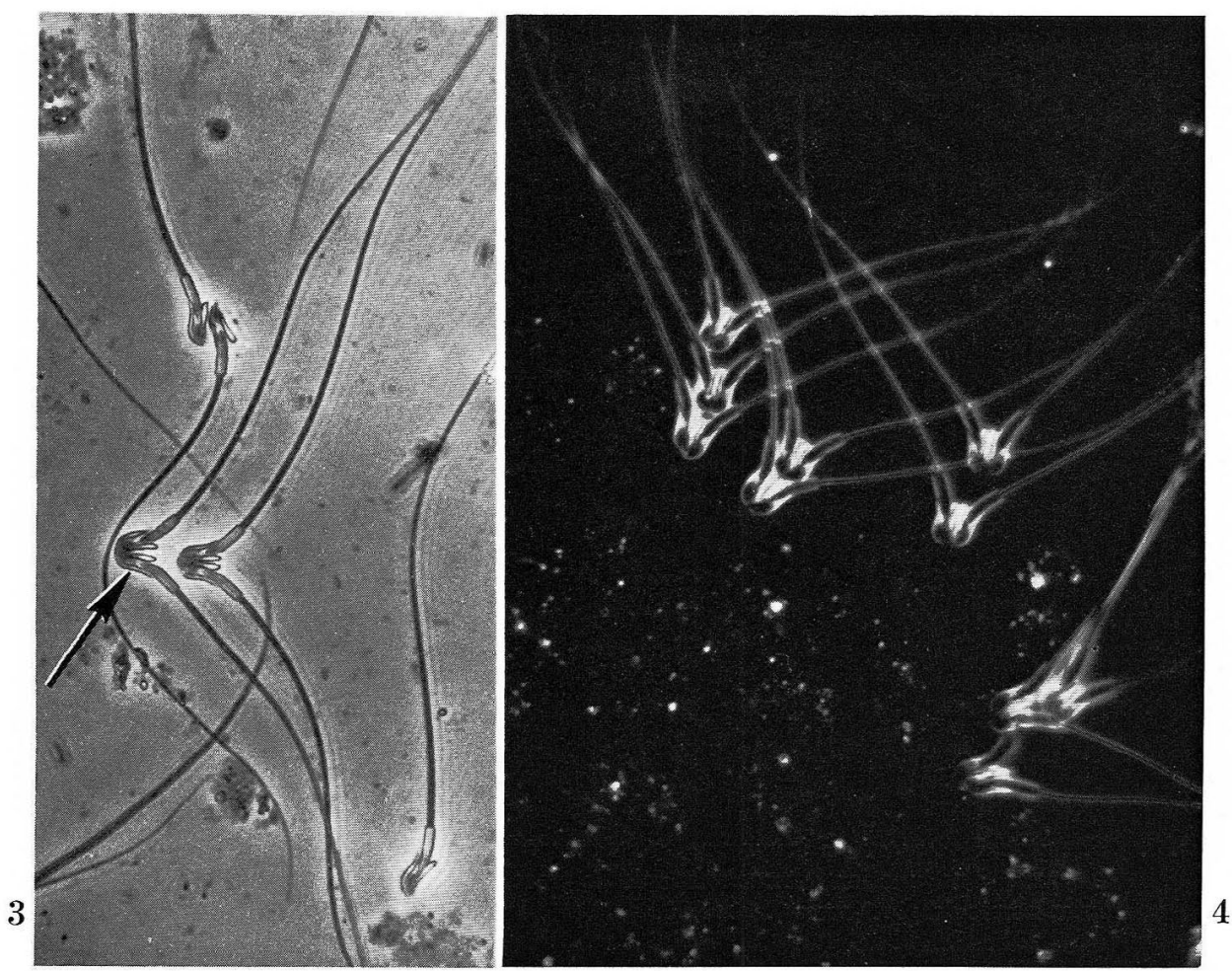

Fig. 3. Seven spermatozoa from the central region of the epididymis. Note that the acrosomal surface of two of the spermatozoa (arrow) are not completely juxtaposed and appear to be in the final stages of forming a pair. Phase contrast. $\times 400$

Fig. 4. All spermatozoa are paired in the caudal region of epididymis. Dark field. $\times 400$

\section{RESULTS}

Spermatozoa from the first portion of the opossum epididymis are characterized by V-shaped nuclei that are positioned at right angles to the long axis of the tail (Fig. 1, 5,6). A prominent cytoplasmic droplet envelops the nucleus and the anterior region of the mid-piece, resulting in the funnel-shaped head of the immature spermatozoon (Fig. 5, 6). An immature acrosome lies on the antero-dorsal surface of the nucleus of the spermatozoa recovered from this region of the epididymis (Fig. 6). All spermatozoa from the first portion of the epididymis exhibit the same general shape and nuclear conformation, are non-motile and remain unpaired.

Spermatozoa from the central region of the epididymis show two marked morphological differences when compared to those in the first portion. A change occurs in the orientation of the nucleus, in which the $\mathrm{V}$-shaped nucleus appears to undergo a $90^{\circ}$ rotation and the nuclear arms come to lie on a plane roughly parallel to the long axis of the tail (Fig. 2,3). The cytoplasmic droplet observed in the immature spermatozoa of the first portion of the epididymis is lost by the time they reach the midportion of the epididymis (Fig. 7). One arm of the V-shaped nucleus appears broader 


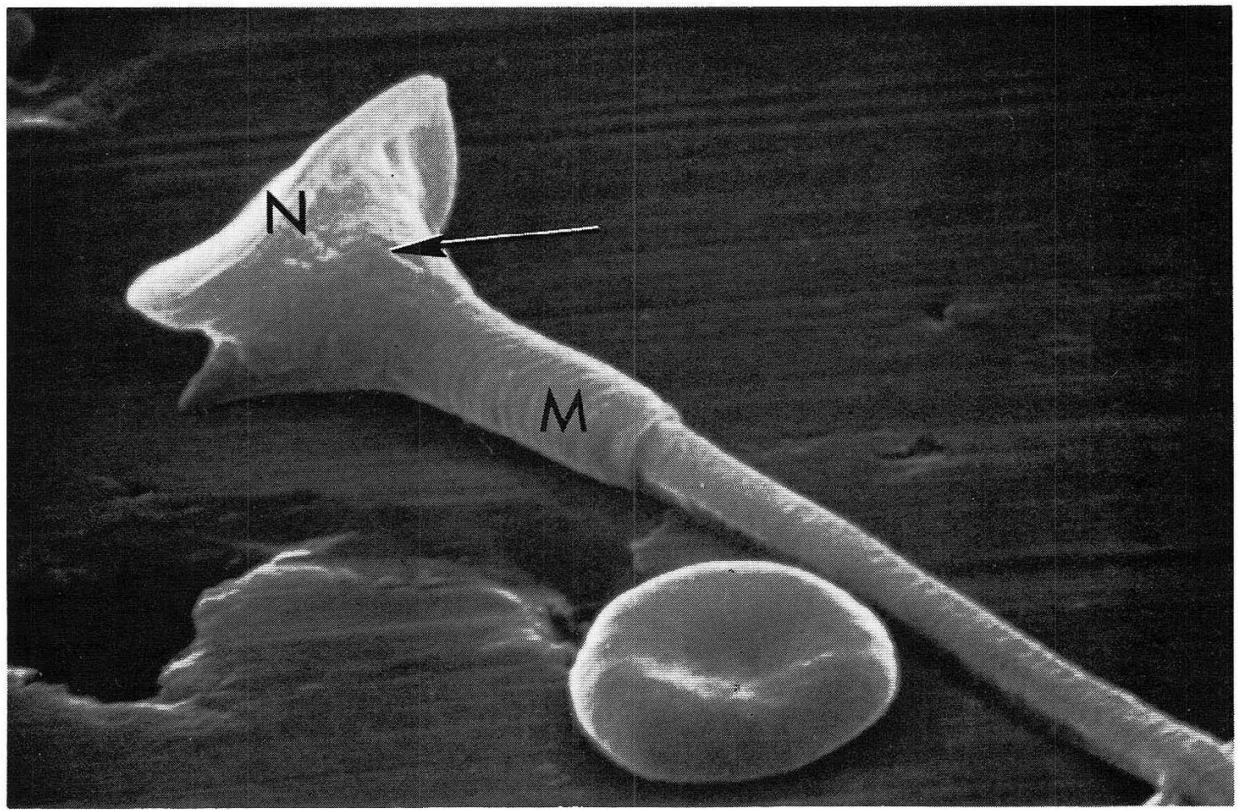

Fig. 5. The caudal view of an immature spermatozoon taken from the first portion of the epididymis. The junction between the middle piece $(M)$ and nucleus $(N)$ is obscured by the cytoplasmic droplet (arrow). $\times 5,000$

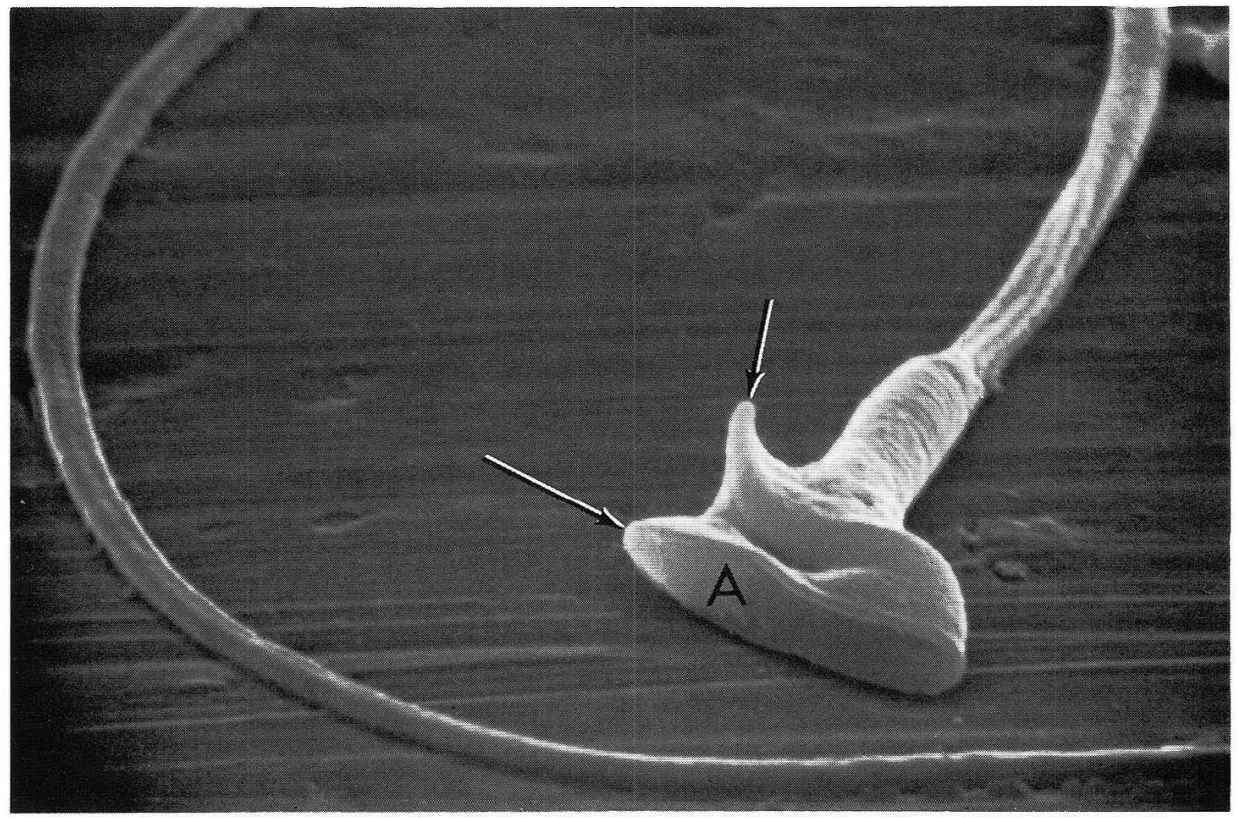

Fig. 6. The cranial view of an immature spermatozoon from the first portion of the epididymis. The two arms of the V-shaped nucleus are clearly shown (arrows). The acrosome $(A)$ lies on the flat surface of the larger arm. Note that the nuclei shown in both Figures 5 and 6 lie on plane perpendicular to the long axis of the tail. $\times 5,000$ 


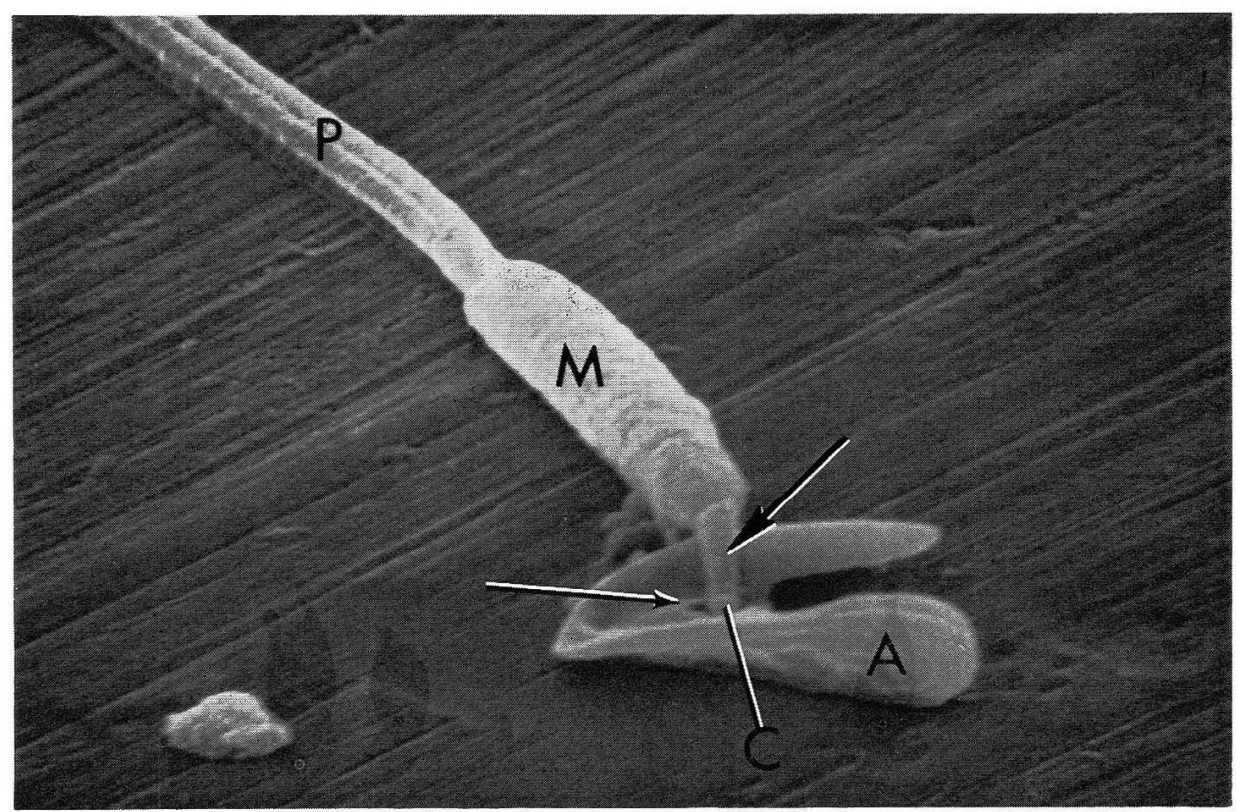

Fig. 7. Partial disruption of a spermatozoon from the central region of the epididymis reveals details of its structural features more clearly. The arms of the $\mathrm{V}$-shaped nucleus from this region normally lie parallel to the long axis of the tail but in this instance the nucleus has been pulled away from the tail. The acrosome $(A)$ lies on the flat surface of the longer nuclear arm. The long connecting piece (large arrow) appears to fit into a concavity (articular fossa) in the nucleus (small arrow). The connecting piece exhibits a distal enlargement representing the capitulum $(C)$. The middle $(M)$ and principal $(P)$ piece of the tail also are shown. $\times 5,000$

and slightly longer than the other arm. The acrosome is borne on the broader arm of the nucleus (Fig. 7). The mid-portion of the tail is attached to the head (nucleus) by a long connecting piece. The rounded end, or capitulum, of the latter fits into the articular fossa of the nucleus (Fig. 7).

Although both paired and unpaired spermatozoa are observed in the mid-region of the epididymis, all show the loss of the cytoplasmic droplet and a re-orientation of the nucleus. Spermatozoa from the central region also show a marked increase in motility.

Virtually all spermatozoa are paired in the caudal region of the epididymis (Fig. 4,8 ). Pairing results from the apposition of the larger nuclear arms and occurs in such a fashion that the flattened acrosomes become sandwiched between the two heads of the juxtaposed spermatozoa (Fig. 9, 10). There is a very close relationship between the adjacent cell membranes. The paired spermatozoa from this region are very active and their tails beat in a coordinated manner. 


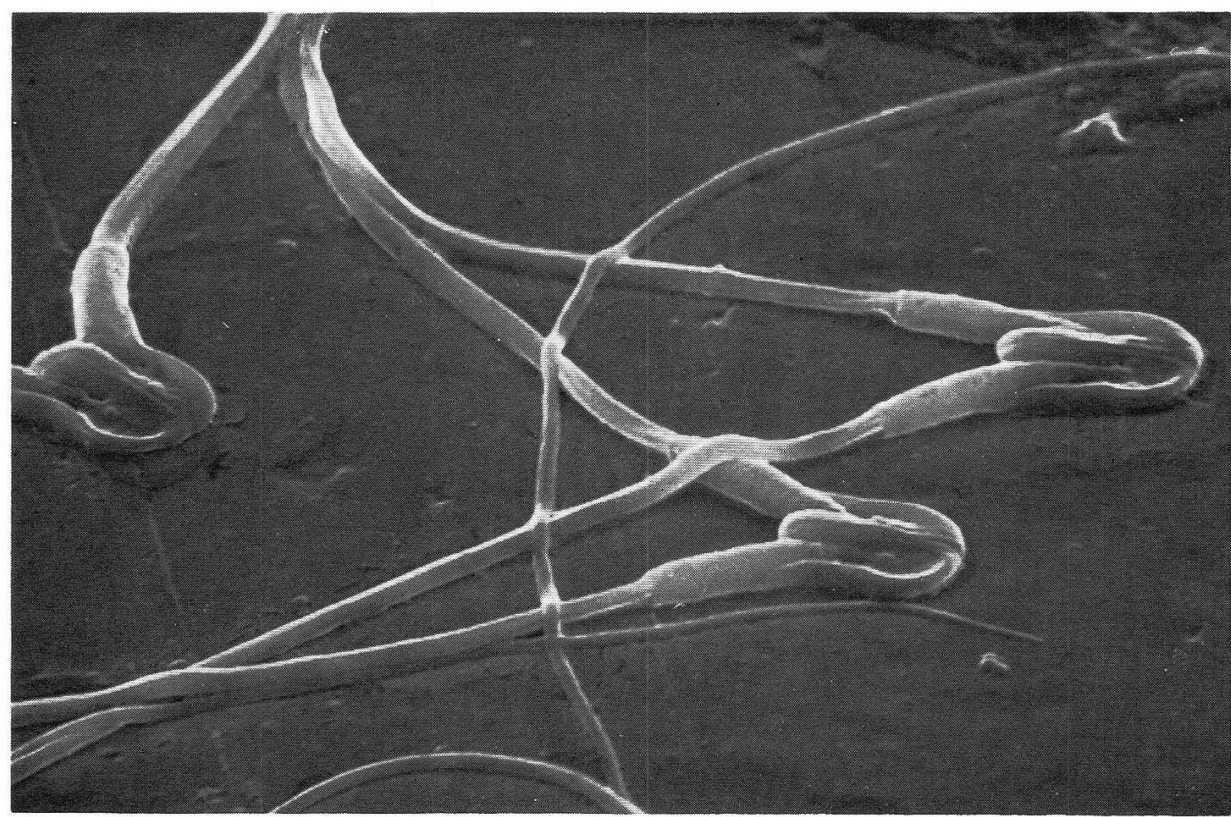

Fig. 8. Three pairs of spermatozoa taken from the caudal region of the epididymis. Note that only the large nuclear arm surfaced by the acrosome participates in the union to form a pair. $\times 2,000$

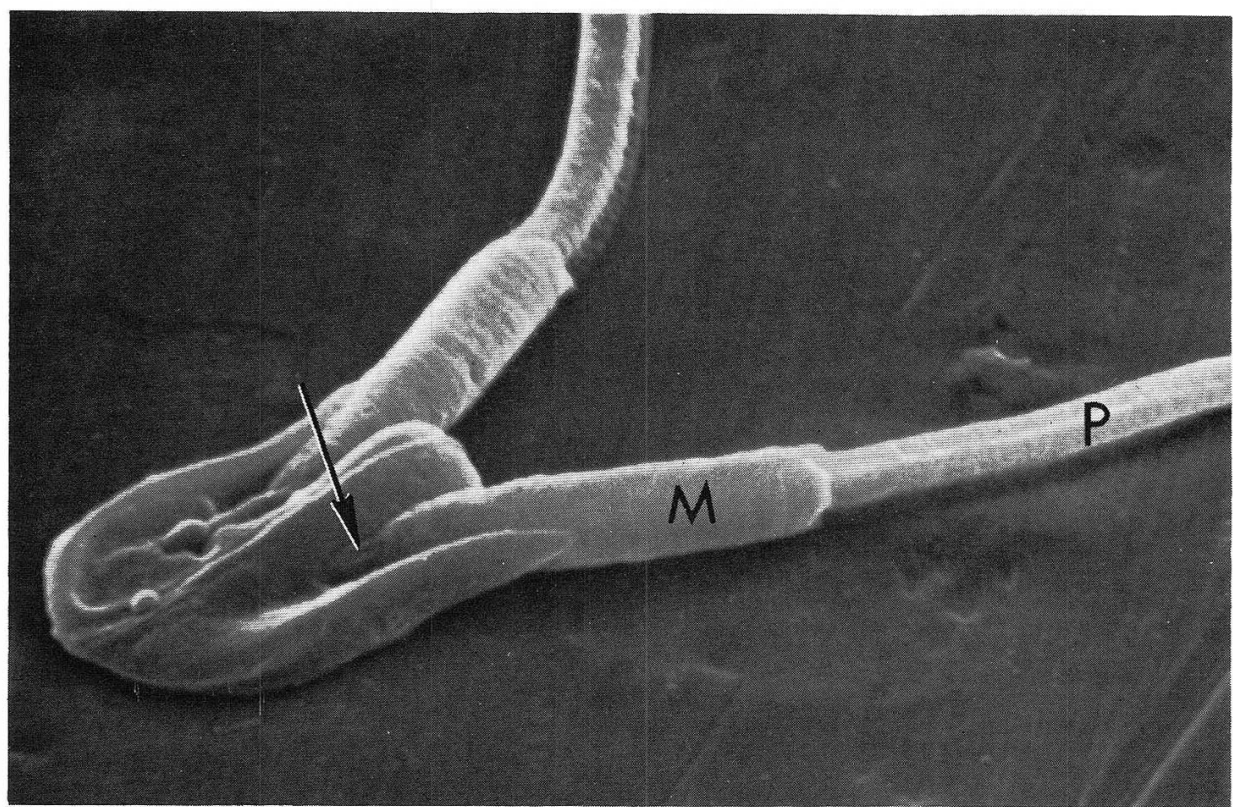

Fig. 9. A frontal view at higher magnification of a sperm pair from the caudal region of the epididymis. Note the V-shaped nucleus exhibiting a large (central) and a small (peripheral) arm. The connecting piece where the tail joins the nucleus is indicated by an arrow. The middle $(M)$ and principal $(P)$ piece of the tail are also seen. $\quad \times 5,000$ 


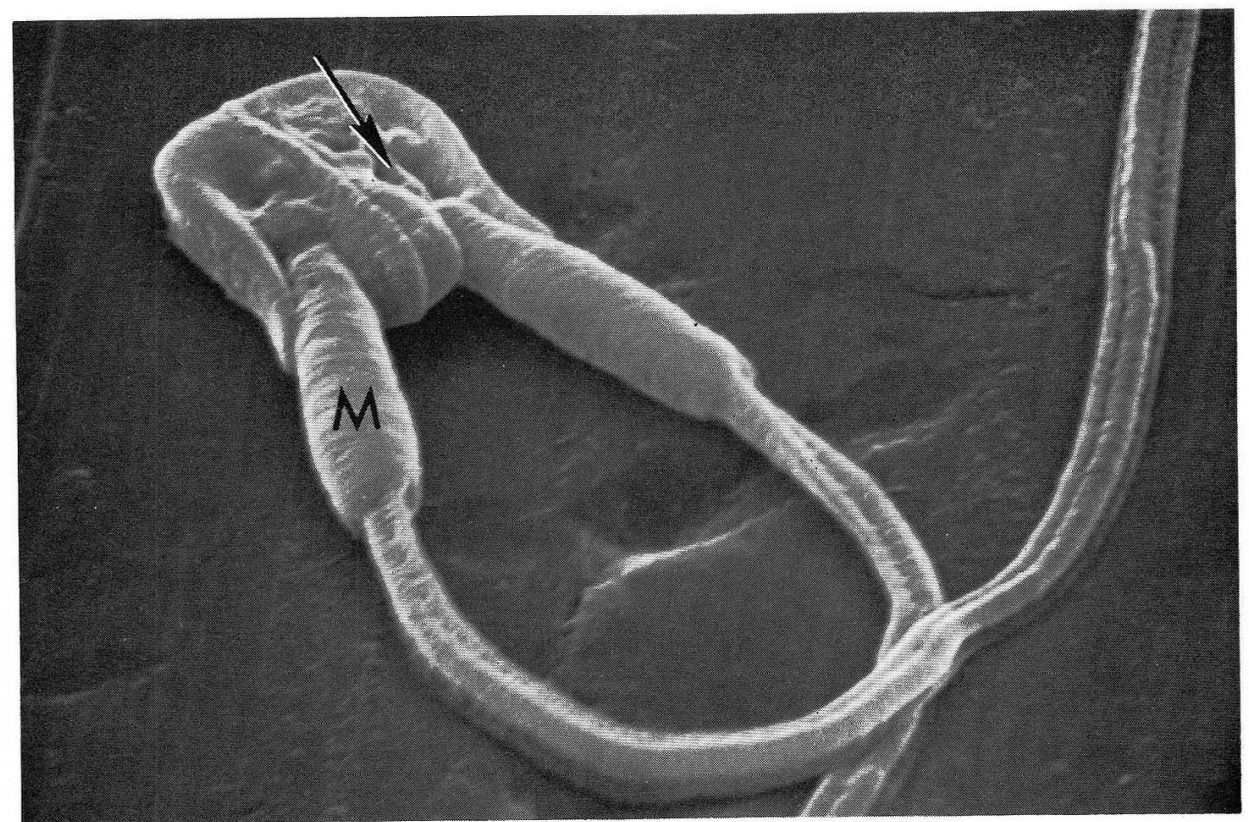

Fig. 10. Paired spermatozoa from the distal portion of the epididymis, viewed from a caudal direction. The V-shaped nucleus, middle piece $(M)$ and connecting piece (arrow) are shown. $\times 5,000$

\section{DISCUSSION}

Although physiological changes (primarily motility) and subtle morphological changes occur during the epididymal maturation of spermatozoa from many eutherian species (Bedford, 1963, 1975; Bedford and Calvin, 1974; Bedford, Calvin and Cooper, 1973; Blandau and Rumery, 1964; Calvin and Bedford, 1971; Fawcett and Hollenberg, 1963; Fawcett and Phillips, 1969; Fray, Hoffer and Fawcett, 1972; Gaddum, 1968), such changes are not nearly as marked as they are in the metatherian forms. The marsupial species are unique in that they exhibit definite changes in morphology (associated with maturation) during their transit through the epididymis. A number of studies have shown that major structural changes occur during the posttesticular maturation of spermatozoa in these particular mammals (HolsteIn, 1965; Phillips, 1970; Harding, Carrick and Shorey, 1975; Temple-Smith and Bedford 1976). The metatherian forms generally show a consolidation of the acrosome, a reorientation of the nucleus and further differentiation of the mid-piece together with a loss of the cytoplasmic droplet. In addition, the pairing of spermatozoa occurs in the epididymis of the new-world forms (Biggers and CreEd, 1962; BigGers and DeLamater, 1965; Phillips, 1970, 1974). Spermatozoa of these animals, like other mammalian forms, become progressively more motile during their passage through the epididymis.

The present scanning electron microscopic study confirms the original transmission electron microscopic observations on immature spermatozoa of Didelphis by 
Holstein (1965) and sperm pairing by Phillips (1974). In the first portion of the epididymis the majority of spermatozoa show a V-shaped nucleus that lies perpendicular to the long axis of the tail. The tail of the spermatozoa of Didelphis is attached to the head by a budlike thickening inserted into an indentation of the nucleus as seen in section (HolsteIn, 1965). We have confirmed this observation with the scanning electron microscope and termed this region of the connecting piece the capitulum and the point where it attaches to the nucleus the articular fossa in accordance with the terminology suggested by FAwcet and Phillips, (1969). A similar attachment between the head and tail is found in the wooly opossum (Caluromys philander) as well (PhIllips, 1970). As spermatozoa pass to the mid-portion of the epididymis the nucleus apparently pivots around this attachment point (the connecting piece) through a $90^{\circ}$ angle so that the arms of the $\mathrm{V}$-shaped nucleus now lie parallel to the long axis of the tail. A similar re-orientation of the nucleus during maturation has been reported in the Australian marsupial Trichosurus vulpecula (Temple-Smith and Bedford, 1976).

Subsequent to the re-orientation of the nucleus pairing occurs between the larger, flat nuclear arms of two spermatozoa sandwiching the acrosomes, which lie on this surface, between the two sperm heads. The only transmission electron micrograph showing the heads of pairing spermatozoa in Didelphis is that of Phillips (1974). The present scanning electron microscopic study not only confirms this description, but also demonstrates that the phenomenon of sperm-pairing in Didelphis occurs regionally in the epididymis.

The present and previous observations on marsupial spermatozoa have shown that in these particular species the spermatozoa show definite morphological changes that can be followed as they pass through the epididymis. Such changes in morphology appear to correspond to the functional maturation of spermatozoa as they become a viable entity during their course through the epididymis. These species should prove to be valuable experimental animals to study not only the phenomenon of sperm maturation but also the role the various segments of the epididymis play in the maturation of spermatozoa. Although the significance of sperm pairing observed in new world marsupials remains unclear, it has been suggested by PHILLIPS (1974) that by pairing the acrosome might be protected during the passage of spermatozoa through the remainder of the male system and in the female tract.

Acknowledgement We are grateful to Dr. J. A. GREen for his help with the phase-contrast and dark-field portion of the study.

\section{REFERENCES}

Arnold, J. D., A. E. Berger and O. L. Allison: Some problems of fixation of selected biological samples for S. E. M. examination. In: Scanning electron microscopy/1971. IIT Research Institute, Chicago, 1971. (Part I, p. 249-256). 
Bedford, J. M.: Changes in the electrophoretic properties of rabbit spermatozoa during passage through the epididymis. Nature (Lond.) 200: 1178-1180 (1963).

-: Maturation, transport and fate of spermatozoa in the epididymis. In: (ed. by) D. W. Hamilton and R. O. Greep: American handbook of physiology, Vol. V, Section 7, Male reproductive system. Waverly Press, Inc., Baltimore, 1975. (p. 303-318).

Bedford, J. M and H. I. Calvin : Changes in the -S-S- linked structures of the sperm tail during epididymal maturation with comparative observations in submammalian species. J. exp. Zool. 187: 181-204 (1974).

Bedford, J. M., H. I. Calvin and G. W. Cooper : The maturation of spermatozoa in the human epididymis. J. Reprod. Fertil., Suppl. 18: 199-213 (1973).

Biggers, J. D. and R. F. S. Creed: Conjugate spermatozoa of the North American opossum. Nature (Lond.) 196: 1112-1113 (1962).

Biggers, J. D. and E. D. DeLamater: Marsupial spermatozoa pairing in the epididymis of American forms. Nature (Lond.) 206: 402-404 (1965).

Blandau, R. J. and R. E. Rumery: The relationship of swimming movements of epididymal spermatozoa to their fertilizing capacity. Fertil. Steril. 15: 571-579 (1964).

Calvin, H. I. and J. M. Bedford: Formation of disulfide bonds in the nucleus and accessory structures of mammalian spermatozoa during maturation in the epididymis. J. Reprod. Fertil., Suppl. 13: 65-75 (1971).

Duesberg, J.: Cytoplasmic structures in the seminal epithelium of the opossum. In: Contributions to embryology. No. 28. Carnegie Institution of Washington, Washington, D. C., 1920.

Fawcett, D. W. and R. D. Hollenberg: Changes in the acrosome of guinea pig spermatozoa during passage through the epididymis. Z. Zellforsch. 60: 276-292 (1963).

Fawcett, D. W. and D. M. Phillips : Observations on the release of spermatozoa and on changes in the head during passage through the epididymis. J. Reprod. Fertil., Suppl. 6: 405-418 (1969).

spermatozoon. Anat. Rec. 165: 153-184 (1969).

Fray, C. S., A. P. Hoffer and D. W. Fawcett: A re-examination of motility patterns of rat epididymal spermatozoa. Anat. Rec. 173: 301-308 (1972).

Gaddum, P.: Sperm maturation in the male reproductive tract: Development of motility. Anat. Rec. 161: 471-482 (1968).

Harding, H. R., F. N. Carrick and C. D. Shorey: Ultrastructural changes in spermatozoa of the brush-tailed possum, Trichosurus vulpecula (Marsupialia) during epididymal transit. Part I: The flagellum. Cell Tiss. Res. 164: 121-132 (1975).

Holstein, A.-F.: Elektronenmikroskopische Untersuchungen am Spermatozoon des Opossum (Didelphy virginiana Kerr). Z. Zellforsch. 65: 904-914 (1965).

Hughes, K. L.: Comparative morphology of spermatozoa from five marsupial families. Austral. J. Zool. 13: 533-543 (1965).

Jordan, H. E.: The spermatogenesis of the opossum (Didelphis virginiana) with special reference to the accessory chromosome and the chondrisomes. Arch. Zellforsch. 7: 41-86 (1911).

McCrady, E.: The embryology of the opossum. In: Amer. Anat. Mem. No. 16. Wistar Institute of Anatomy and Biology, Philadelphia, 1938. (p. 1-226).

Phillips, D. M. : Ultrastructure of spermatozoa of the wooly opossum, Caluromys philander. J. Ultrastr. Res. 33: 381-397 (1970).

- : Comparative analysis of mammalian sperm motility. J. Cell Biol. 53: 561-573 (1972).

-: Spermiogenesis. Academic Press, New York, 1974.

Retzius, G.: Die Spermien von Didelphys. Biol. Untersuch. 14: 123-126 (1909).

Selenka, E.: Studien über Entwickelungsgeschichte der Thiere. 4. Ht. Das Opossum (Didelphys virginiana). C. W. Kreidel's Verlag, Wiesbaden, 1887.

Temple-Smith, P. D. and J. M. Bedford: The features of sperm maturation in the epididymis 
of a marsupial, the brushtailed opossum Trichosurus vulpecula. Amer. J. Anat. 147: 471-500 (1976).

William J. Krause, Ph. D. Department of Anatomy School of Medicine University of Missouri Columbia, Missouri 65212 U.S. A. 